\title{
Prospective registry validating the reproducibility of mitral paravalvular leak measurements in a standardized real-time three-dimensional transesophageal echocardiography algorithm for optimal choice of the closure device
}

\author{
Piotr Pysz, Michał Kozłowski, Magdalena Malczewska, Eliza Adamczyk-Filipek, Wojciech Wojakowski, \\ Grzegorz Smolka
}

Department of Cardiology and Structural Heart Diseases, School of Medicine in Katowice, Medical University of Silesia, Katowice, Poland

Adv Interv Cardiol 2019; 15, 2 (56): 203-210

DOI: https://doi.org/10.5114/aic.2019.86013

\begin{abstract}
A bstract
Introduction: Transcatheter paravalvular leak closure (TPVLC) has become a well-established method of treatment for patients with paravalvular leak (PVL) causing heart failure or significant hemolysis. Nonetheless, the method of optimal PVL sizing and subsequent device choice requires standardization. For this purpose a real-time three-dimensional transesophageal echocardiography (RT-3D TEE) algorithm was developed in our institution. It has proven clinically useful with results successfully translated into type, size, and number of occluding devices. Still, the reproducibility of measurements has not been previously verified.

Aim: To investigate the intra- and inter-observer variability of measurements in a RT-3D TEE algorithm developed for optimal choice of occluding devices during TPVLC.

Material and methods: Three echocardiographers, with RT-3D TEE clinical experience ranging from 1 to 8 years, analyzed recordings of 20 mitral PVLs according to our standardized protocol. PVL channel cross-sectional area (CSA), width (W) and length (L) were measured at the level of the vena contracta. Each echocardiographer performed the measurements twice on different days, individually and blinded to other participants' results.

Results: Measurements of PVL CSA, W and L showed intra- and interobserver agreement of $0.98,0.93,0.92$ and $0.95,0.88,0.87$, respectively.

Conclusions: The presented algorithm enables standardized utilization of RT-3D TEE for appropriate TPVLC device choice with low intra- and inter-observer variability.
\end{abstract}

Key words: paravalvular leak device closure, real-time three-dimensional transesophageal echocardiography, measurement standardization.

Su m m a ry

Our study aimed to investigate the reproducibility of mitral paravalvular leak (PVL) measurements in a standardized real-time three-dimensional transesophageal echocardiography algorithm developed for optimal choice of occluding devices during transcatheter paravalvular leak closure. Values of PVL channel cross-sectional area, width and length, obtained independently by three echocardiographers with different RT-3D TEE clinical experience, showed low intra- and inter-observer variability. 


\section{Introduction}

Paravalvular leaks (PVL) after surgical valve replacement are reported in more than $10 \%$ of patients $[1,2]$. The first transcatheter PVL closure (TPLVC) was performed over two decades ago [3] and the method has been constantly developing ever since. Having finally been shown to improve the functional class of heart failure patients and reduce hemolysis [4] it was granted a class Ila recommendation by the AHA/ACC [5]. Initially, TPVLC was performed with ASD, VSD or PDA occluders and later with plugs designed for vascular embolization such as Amplatzer Vascular Plugs (AVP) [6-9]. The first TPVLC-dedicated device to receive the CE mark is the Paravalvular Leak Device, PLD (Occlutech).

Ultrasound imaging, with real-time three-dimensional transesophageal echocardiography (RT-3D TEE) in particular, is an extremely useful tool for procedural guiding during TPVLC [10]. Likewise, it may play a crucial role in optimal, anatomy-matched, choice of type, size, and number of the occluding devices. To optimize TPVLC device selection, we developed a novel, standardized protocol of TEE image acquisition and analysis. Applicability of our approach was validated clinically, with a procedural success rate ranging from $89.7 \%$ to $93.8 \%$ depending on the device $[9,11,12]$.

\section{Aim}

Until now the described TEE protocol was used in our center almost exclusively by one experienced echocardiographer - hence the intra- and inter-observer variability of the method remained unknown. We currently intend to determine these parameters for investigators with varying experience in 3D-RT TEE.

\section{Material and methods}

\section{D TEE protocol}

To ensure optimal spatial and temporal resolution, we acquire the smallest possible volumes containing the complete PVL channel in an ECG-gated, single-beat zoom 3D High Volume Rate (HVR) mode with color doppler (CD). Such visualization of PVL opacification by CD-mapped flow turbulence is used for detailed PVL anatomy analysis. With acquired volume being replayed in the multiplanar presentation the PVL channel is reoriented within the three perpendicular planes until two of them are positioned parallel to the flow direction while the third one is perpendicular and at the level of the vena contracta (VC) - Figure 1. Pivotal for the device choice are the cross-sectional area (CSA) and the minimum (width $-W$ ) and maximum (length $-\mathrm{L}$ ) diameters of $\mathrm{VC}$, all measured

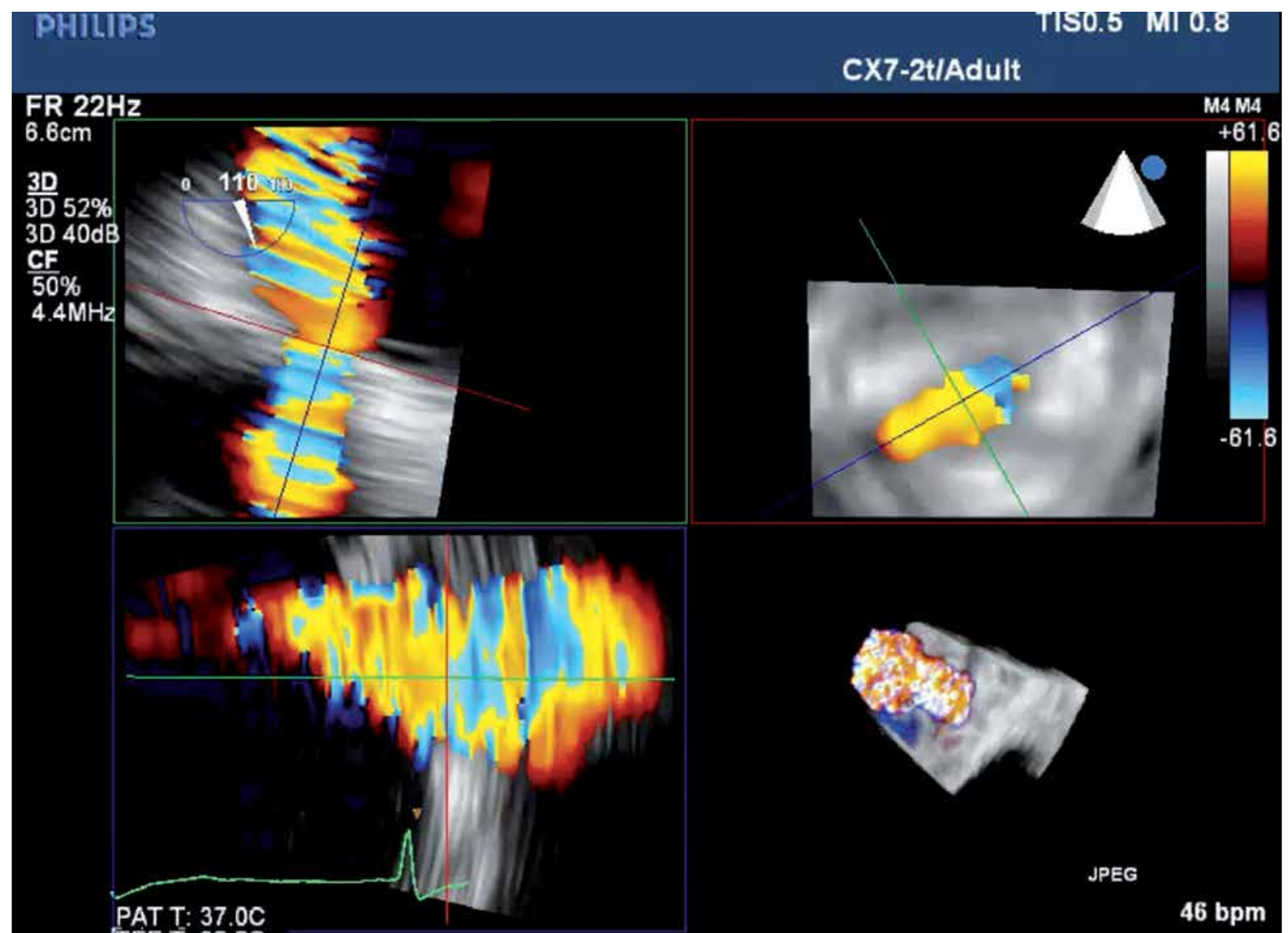

Figure 1. Multiplanar presentation focused on PVL VC visualization 

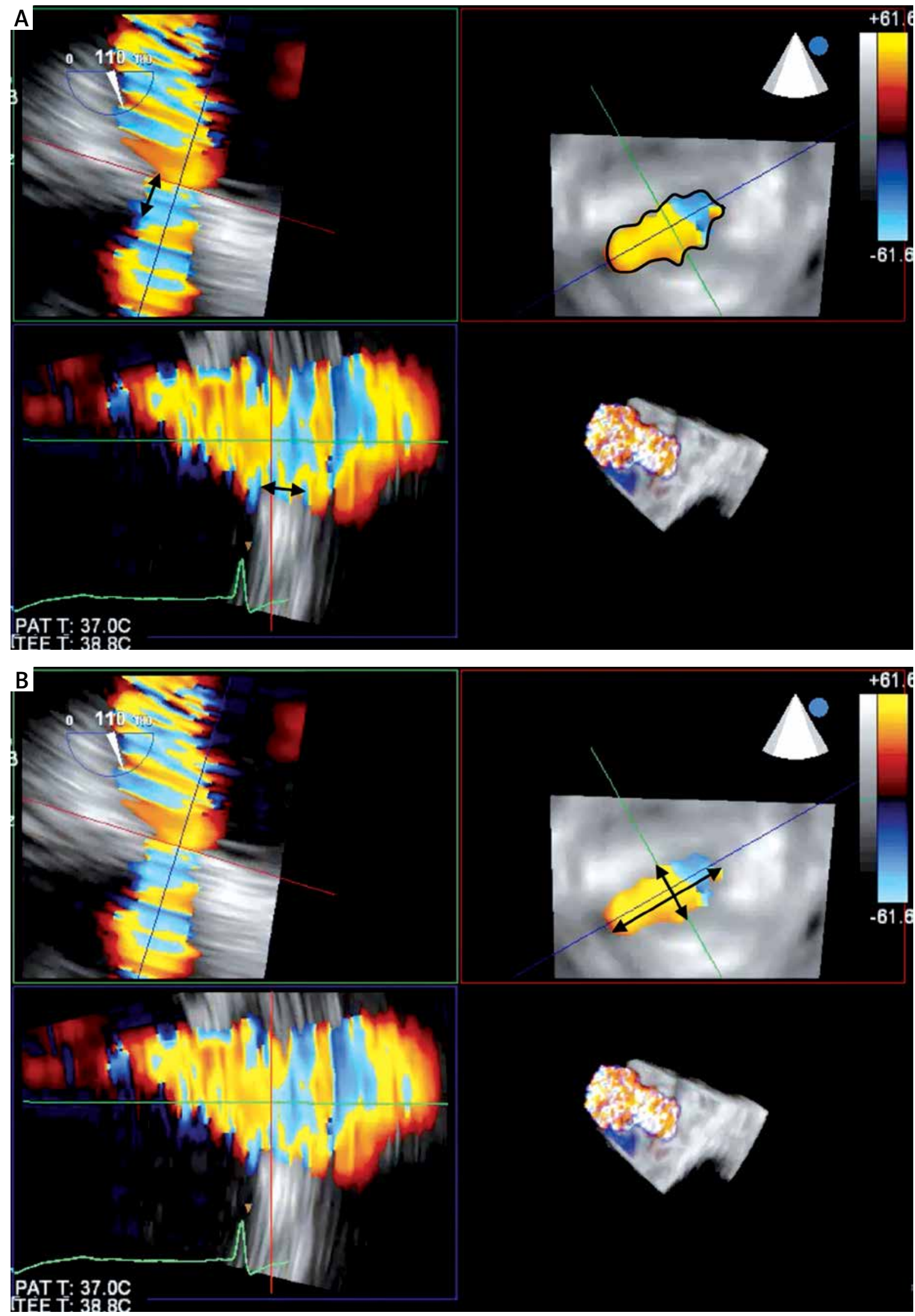

Figure 2. A - Measurements of PVL VC CSA (black continuous trace) and depth (black double-headed arrows), B - Measurements of PVL minimum (width) and maximum (length) dimensions (black double-headed arrows) 
on the image frozen at the time of maximum paravalvular backflow. Analogously, the channel depth, understood as the distance between cavities of the left ventricle (LV) and left atrium (LA), can be verified (Figures $2 \mathrm{~A}$ and $B$ ). The size and number of plugs are chosen in previously described manners according to either CSA for AVP III $[9,11]$ or W and L for PLD [12].

\section{Validation of measurements' reproducibility}

Three researchers (R1, R2, R3) with different levels of 3D-RT TEE expertise (8, 3 and 1 year of clinical experience, respectively) were chosen to participate in the study. Recordings of 20 patients with mitral PVLs referred for TPVLC were acquired in the above-described manner using Philips iE33 and EPIQ 7 ultrasound machines (Philips). Heart failure symptoms in functional NYHA class $\geq 3$ ( $n=19,95 \%)$ constituted the main indication for TPVLC. Some extent of concomitant hemolytic anemia was present in 13 (65\%) patients but did not require RBC transfusion. One patient was disqualified following the TEE and clinical assessment, as the PVL was deemed insignificant. The group charac- teristics are presented in Table I. 3D HVR loops with CD were analyzed offline on a workstation (QLAB Ultrasound Cardiac Analysis, Philips). All researchers were asked to follow the same steps: 1) determine the location of the $P V L$ and choose a frame during systole in which the PVL is best visible, 2) align the multiplanar reconstruction planes to obtain the cross-sectional image of the PVL channel at the level of the VC (representing the "landing zone" for closure device), 3) measure the W, L and CSA of PVL at that level. Each echocardiographer individually performed the measurements twice on different days without knowledge of values obtained by other participants. The echocardiographer with the shortest experience in 3D echocardiography was introduced to the principles of PVL measurements with analysis of three cases (not included in the study).

\section{Results}

Intra- and interobserver correlation coefficients were calculated for each measurement (CSA, W, and L). All of them were found to be highly reproducible. Results are presented in Table II. Bland and Altman showing in-

Table I. Clinical and procedural group characteristics

\begin{tabular}{|c|c|c|c|c|c|c|c|c|c|c|c|c|}
\hline Age & Sex & $\mathrm{HF}$ & Hem & $\mathrm{AF}$ & $\mathrm{HA}$ & $\mathrm{DM}$ & CKD & Prosth & Occl & Size & Num & Res \\
\hline 59 & $M$ & 1 & 0 & 2 & 0 & 0 & 0 & Biol & PLD & $4 \times 2$ & 1 & 0 \\
\hline 80 & $\mathrm{~F}$ & 1 & 1 & 2 & 0 & 0 & 1 & Mech & PLD & $8 \times 4$ & 1 & 0 \\
\hline 59 & $F$ & 1 & 0 & 2 & 0 & 0 & 1 & Biol & PLD & $12 \times 5$ & 1 & 0 \\
\hline 59 & $\mathrm{~F}$ & 1 & 1 & 1 & 1 & 1 & 0 & Mech & PLD & $8 \times 4$ & 1 & 1 \\
\hline 75 & $M$ & 1 & 0 & 0 & 1 & 0 & 0 & Biol & PLD & $6 \times 3$ & 1 & 0 \\
\hline 67 & $M$ & 1 & 0 & 2 & 0 & 0 & 1 & Biol & PLD & $16 \times 8$ & 1 & 0 \\
\hline 66 & $M$ & 1 & 0 & & 1 & 0 & 0 & Mech & PLD & $8 \times 4$ & 1 & 0 \\
\hline 75 & $M$ & 1 & 1 & 1 & 1 & 0 & 0 & Biol & PLD & $12 \times 5$ & 1 & 1 \\
\hline 60 & $\mathrm{~F}$ & 1 & 1 & 2 & 0 & 0 & 0 & Mech & AVP III & $3 \times 6$ & 3 & 1 \\
\hline 58 & $F$ & 1 & 1 & 2 & 0 & 1 & 0 & Biol & PLD & $5 \times 5$ & 1 & 1 \\
\hline 73 & $\mathrm{~F}$ & 1 & 1 & 0 & 0 & 0 & 1 & Mech & AVP III & $6 \times 3$ & 3 & 1 \\
\hline 84 & $F$ & 1 & 1 & 2 & 1 & 0 & 1 & Biol & PLD & $12 \times 5$ & 1 & 1 \\
\hline 65 & $M$ & 1 & 1 & 1 & 1 & 0 & 0 & Mech & AVP III & $6 \times 3$ & 4 & 1 \\
\hline 67 & $M$ & 1 & 1 & 2 & 1 & 0 & 1 & Mech & AVP III & $6 \times 3$ & 4 & 0 \\
\hline 55 & $M$ & 1 & 0 & 0 & 0 & 0 & 0 & Mech & AVP III & $6 \times 3$ & 4 & 1 \\
\hline 65 & $F$ & 1 & 1 & 2 & 0 & 0 & 0 & Biol & ASD & 12 & 1 & 1 \\
\hline 70 & $\mathrm{~F}$ & 1 & 1 & 0 & 0 & 0 & 0 & Biol & AVP III & $6 \times 3,8 \times 4$ & $3+1$ & 0 \\
\hline 73 & $M$ & 1 & 1 & 2 & 1 & 1 & 1 & Mech & AVP III & $6 \times 3$ & 3 & 0 \\
\hline 65 & $F$ & 1 & 1 & 0 & 0 & 0 & 0 & Mech & PLD & $8 \times 4$ & 1 & 0 \\
\hline 59 & $M$ & 0 & 0 & 0 & 1 & 0 & 0 & Biol & NA & NA & NA & NA \\
\hline
\end{tabular}

HF-heart failure symptoms $\geq$ NYHA class III, Hem-hemolytic anemia, AF-atrial fibrillation (1-paroxysmal, 2 - permanent), HA - arterial hypertension, DM - type 2 diabetes mellitus, CKD - chronic kidney disease stage 3 or 4, Prosth - type of prosthetic valve, Ocd - type of implanted PVL occluders, Size - size of implanted PVL occluders, Num - number of implanted PVL occluders, Res - grade of residual PVL (O - none, 1 -trace/mild). 
tra-observer (R1, R2, R3) differences in CSA, W and L measurements are presented in Figure 3. Bland and Altman plots showing inter-observer differences for each of the CSA, $W$ and $L$ measurements are presented in Figure 4. The limits of agreement (LoA), defined as the mean difference \pm 1.96 SD of differences, were not exceeded in the vast majority of measurements.

\section{Discussion}

Our method of mitral PVL sizing aims at proper closure device selection. Excellent clinical applicability has already been reported before but in the majority of cases only one, experienced echocardiographer performed all measurements [9-12]. Current analysis shows that the proposed algorithm offers excellent both intra-observer reproducibility and interobserver agreement even if used by echocardiographers with very different levels of 3D-RT TEE expertise.

The described algorithm is not meant to assess the hemodynamic significance of the PVL, which typically requires careful analysis of both echocardiographic pa-
Table II. Intraclass correlation coefficients (ICC) for measurements of PVL vena contracta crosssectional area (CSA), width and length

\begin{tabular}{lcc} 
Parameter & $\begin{array}{c}\text { Interobserver ICC } \\
\text { Median }(95 \% \mathrm{CI})\end{array}$ & $\begin{array}{c}\text { Intraobserver ICC } \\
\text { Median }(95 \% \mathrm{CI})\end{array}$ \\
\hline CSA & $0.95(0.89-0.98)$ & $0.98(0.94-0.99)$ \\
\hline Width & $0.88(0.77-0.95)$ & $0.93(0.84-0.97)$ \\
\hline Length & $0.87(0.76-0.94)$ & $0.92(0.79-0.97)$
\end{tabular}

rameters and clinical status of the patient. Nevertheless, it may be implemented for TPVLC effect assessment [13].

Assuring sufficient quality of acquired volume data sets is of paramount importance for the presented algorithm of analysis. Essentially, the ultrasound window in the middle transesophageal view is excellent in nearly all patients with mitral PVL. Nevertheless, standard 3D full volume with color Doppler (CD) acquisition, while tremendously useful for determining PVL location, does not allow for detailed PVL channel visualization as large vol-
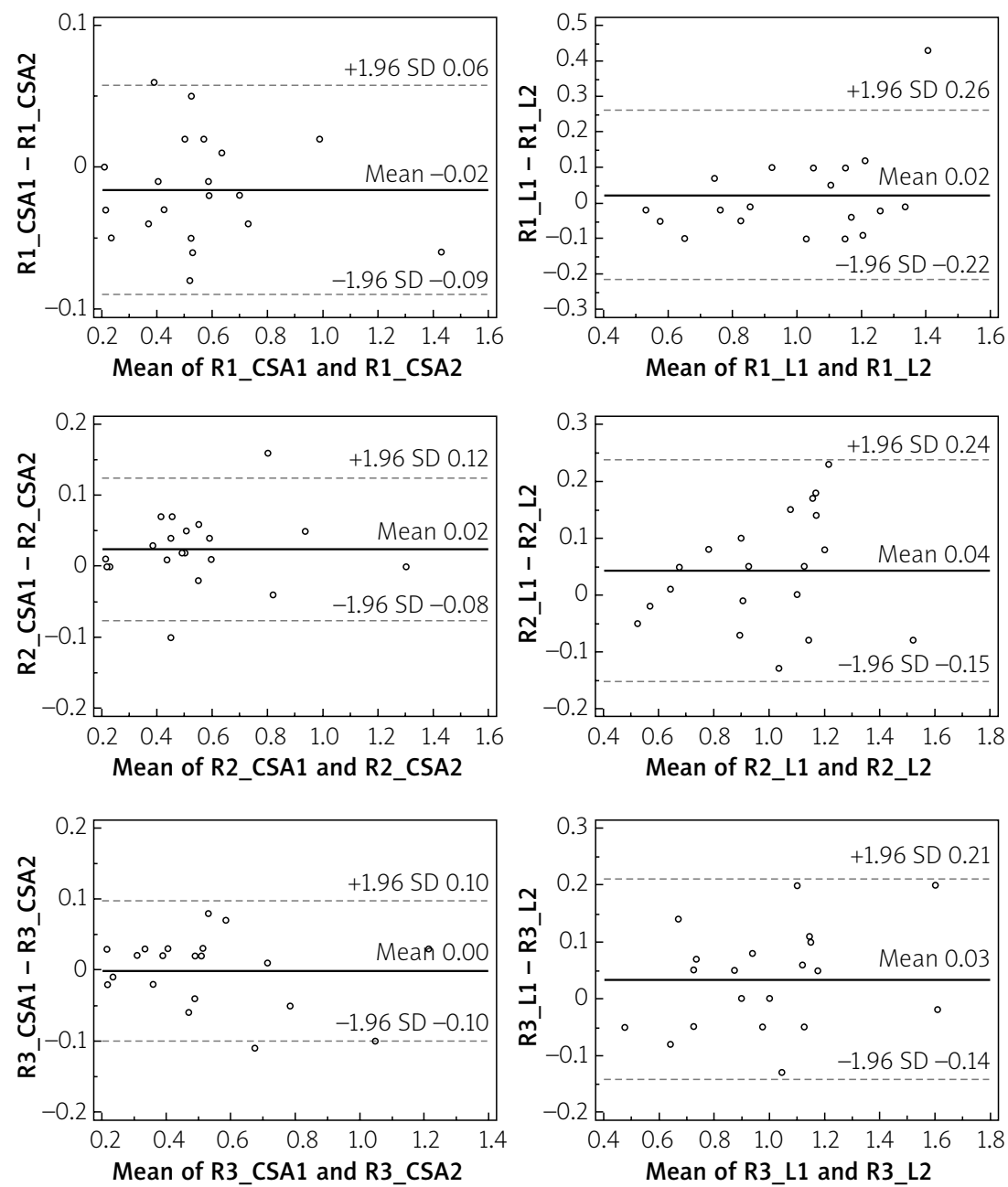
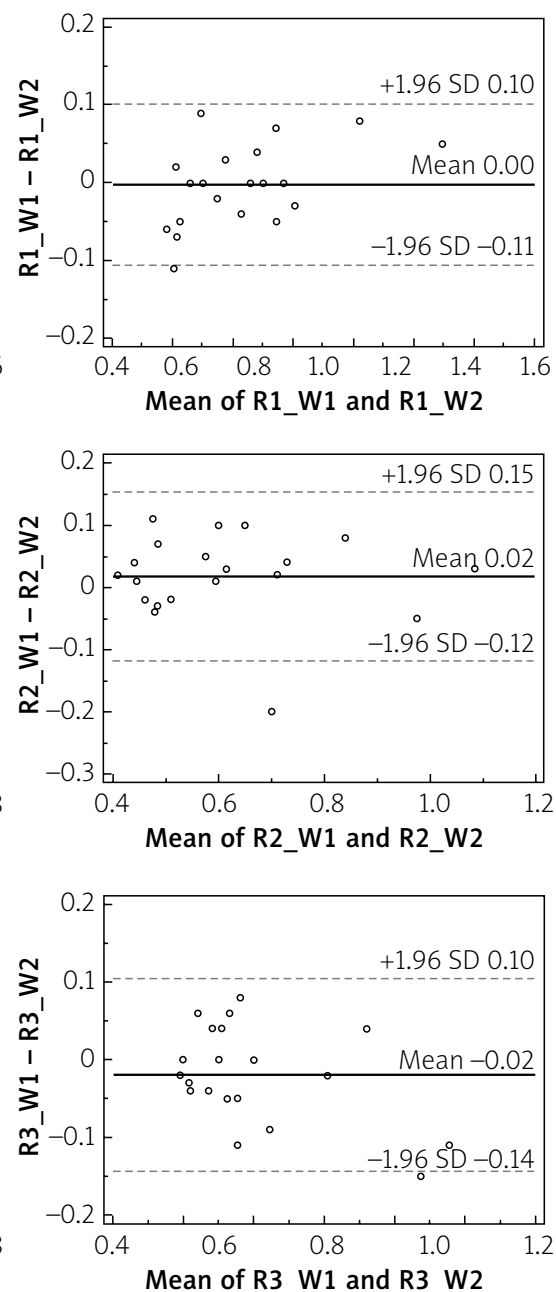

Figure 3. Bland and Altman plots presenting intra-observer differences in cross-sectional area (CSA), width (W) and length (L) measurements 

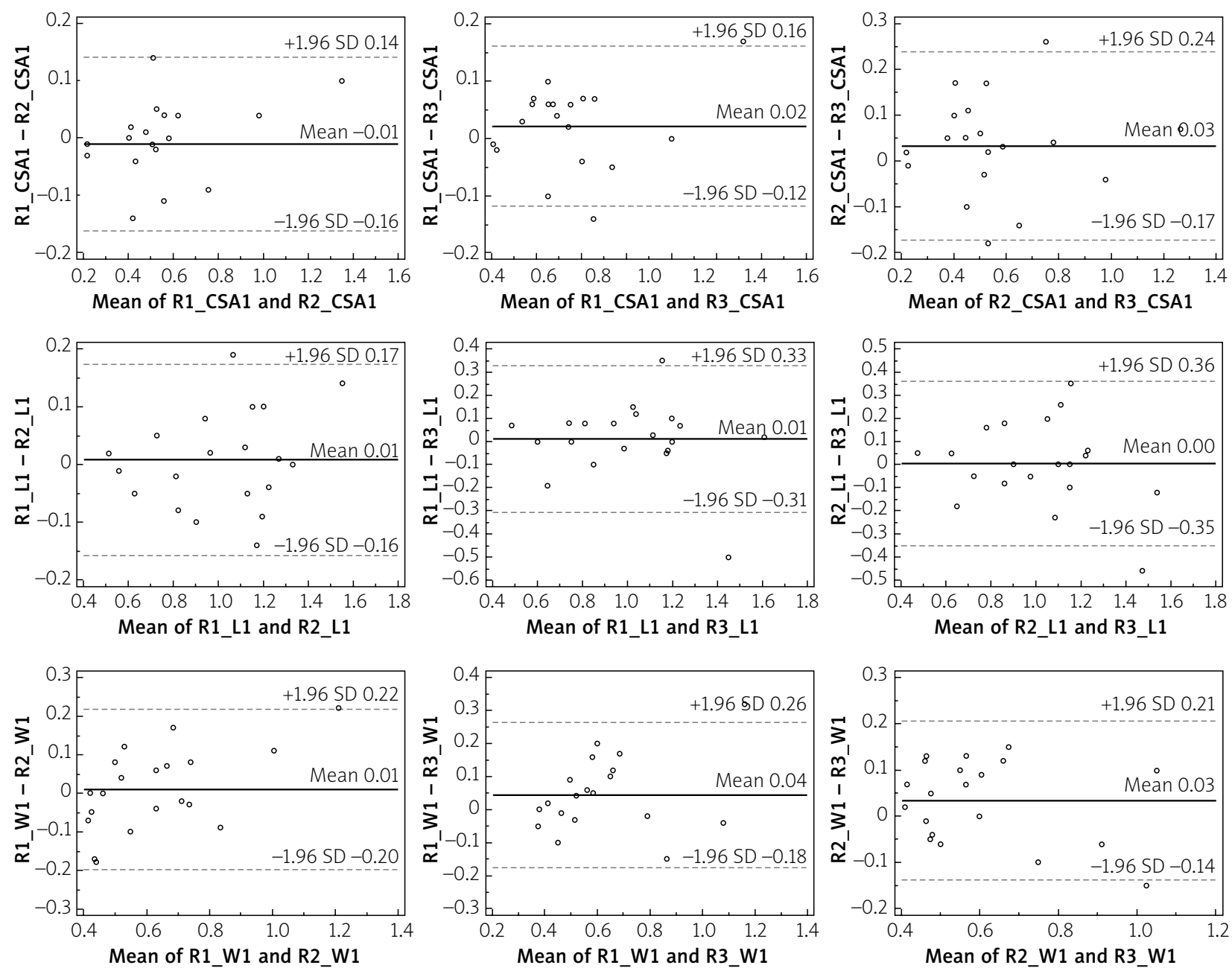

Figure 4. Bland and Altman plots presenting inter-observer differences for each of the cross-sectional area (CSA), width (W) and length (L) measurements

ume per se reduces the resolution. Merging the sectors in multi-beat ECG-gated acquisition mode, while enhancing the volume rate, may also produce stitching artifacts, particularly in the settings of atrial fibrillation. As a result, the image quality can easily become non-diagnostic unless the presented acquisition mode is followed.

The idea behind the presented imaging scheme is to facilitate selection of the device(s) leading to complete PVL sealing with the smallest possible amount of occluders' material left outside the PVL channel. The need of possibly total PVL closure is not only justified by the intuitively optimal hemodynamic result but also by avoiding hemolysis exacerbation, which may occur if only partial reduction of paravalvular flow is achieved [13]. In our center, visualization of an irregularly shaped and/or long PVL channel justifies the use of multiple AVP III occluders (devices containing no sealing fabric) with the intention to densely fill the PVL channel with squeezed Nitinol mesh (oversizing is desired). With PLD, on the other hand, the distal discs contain fabric, and therefore their full expansion and apposition to PVL orifices are needed. To achieve that, the middle module of the devices should not be squeezed within the channel (oversizing contraindicated) as it results in deformation of the whole device (Figures $5 \mathrm{~A}, \mathrm{~B}$ ). One of the hereby-analyzed patients was finally treated with ASD implantation as the PVL was located relatively far from the prosthesis and the channel was large and elongated. In such large lesions, the PVL channel can also be sometimes directly visualized without CD mapping. In our experience, directly measured dimensions tend to be slightly smaller when compared to those visualized with $C D$, but this technique is only feasible in selected patients and lacks sufficient clinical validation for TPVLC.

Avoidance of overhanging of the devices outside the lesion reduces the risk of prosthetic valve discs impingement. Another risk related to the excess of artificial material left protruding from the channel is that of thromboembolism. Current evidence on the topic is scarce, but published case reports point to either lack or incompleteness of device endothelialization. Follow-up in these observations reaches up to 16 months, and thrombus formation on the closing device has also been reported [14]. 

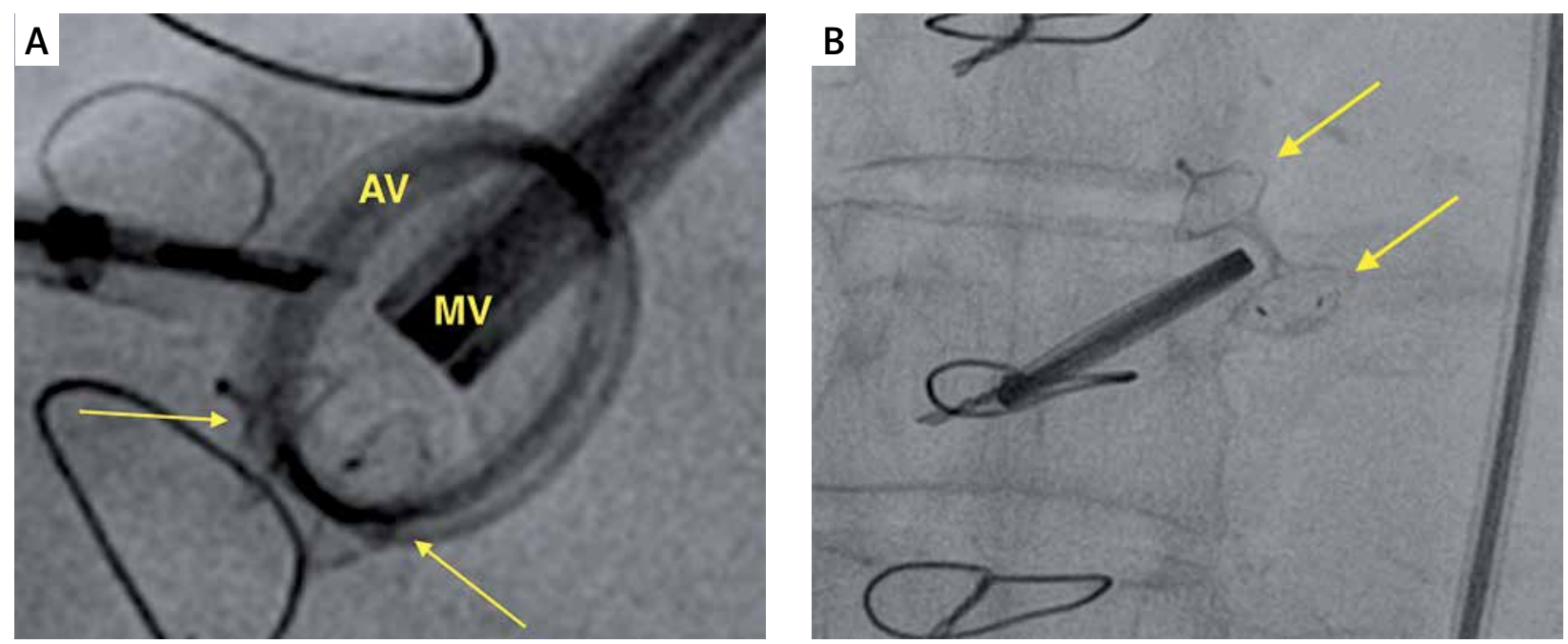

Figure 5. A - Fluoroscopy image of a properly expanded PLD (PLD closing mitral PVL, arrows pointing at the discs of PLD). B - Fluoroscopy image of a deformed PLD (arrows pointing at the discs of PLD) $M V$ - mitral prosthetic valve, AV-aortic prosthetic valve.

Indirectness of the proposed method of PVL channel sizing, based solely on paravalvular backflow measurements visualized by color-coded pulse wave Doppler, may be considered a limitation of our method. The high-velocity flow of blood at the level of the PVL VC produces numerous turbulences by $C D$ and does not necessarily represent actual anatomical dimensions of the PVL channel. Still, this approach has been shown to be very useful in real-life, clinical situations [9-13].

Secondly, even though our 3 participants performed measurements individually, they were all working on images (volume loops) acquired by the most experienced one. Obviously, image quality is pivotal for our algorithm, and high technical skills of the echocardiographer performing the examination are mandatory for final success.

\section{Conclusions}

The presented algorithm had been previously reported to facilitate standardized utilization of RT-3D TEE for adequate PVL sizing and TPVLC device choice, if used by a highly experienced echocardiographer. We currently found that it can also be efficiently used by echocardiographers at different proficiency levels with low intra- and inter-observer variability.

\section{Acknowledgments}

The study was supported by the STRATEGMED ॥ grant (National Centre for Research and Development, STRATEGMED2/269488/7/NCBR/2015). The results were presented as an abstract during the $22^{\text {nd }}$ International Congress of the Polish Cardiac Society.

\section{Conflict of interest}

The authors declare no conflict of interest.

\section{References}

1. Davila-Roman VG, Waggoner AD, Kennard ED, et al. Prevalence and severity of paravalvular regurgitation in the Artificial Valve Endocarditis Reduction Trial (AVERT) echocardiography study. J Am Coll Cardiol 2004; 44: 1467-72.

2. Hwang HY, Choi JW, Kim HK et al. Paravalvular leak after mitral valve replacement: 20-year follow-up. Ann Thorac Surg 2015; 100: $1347-52$.

3. Hourihan M, Perry SB, Mandell VS, et al. Transcatheter umbrella closure of valvular and paravalvular leaks. J Am Coll Cardiol 1992; 20: 1371-7.

4. Millan X, Skaf S, Joseph L, et al. Transcatheter reduction of paravalvular leaks: a systematic review and meta-analysis. Can J Cardiol 2015; 31: 260-9.

5. Nishimura RA, Otto CM, Bonow RO, et al. 2014 AHA/ACC guideline for the management of patients with valvular heart disease: executive summary: a report of the American College of Cardiology/American Heart Association Task Force on Practice Guidelines. J Am Coll Cardiol 2014; 63: 2438-88.

6. Nietlispach F, Johnson M, Moss RR, et al. Transcatheter closure of paravalvular defects using a purpose-specific occluder. JACC Cardiovasc Interv 2010; 3: 759-65.

7. Cruz-Gonzalez I, Rama-Merchan JC, Arribas-Jimenez A, et al. Paravalvular leak closure with the Amplatzer Vascular Plug III device: immediate and short-term results. Rev Esp Cardiol (Engl Ed) 2014; 67: 608-14.

8. Fiszer R, Smolka G, Szkutnik M, et al. Transcatheter aortic paravalvular leak closure using 3 Amplatzer Vascular Plug III devices in a child. Postep Kardiol Inter 2015; 11: 156-7.

9. Smolka G, Pysz P, Jasinski M, et al. Multiplug paravalvular leak closure using Amplatzer Vascular Plugs III: a prospective registry. Catheter Cardiovasc Interv 2016; 87: 478-87.

10. Zamorano J, Badano LP, Bruce C, et al. EAE/ASE recommendations for the use of echocardiography in new transcatheter interventions for valvular heart disease. Eur Heart J 2011; 32: 2189-214.

11. Smolka G, Pysz P, Jasinski M, et al. Transapical closure of mitral paravalvular leaks with use of Amplatzer vascular plug III. J Invasive Cardiol 2013; 25: 497-501. 
12. Smolka G, Pysz P, Kozłowski M, et al. Transcatheter closure of paravalvular leaks using a paravalvular leak device - a prospective Polish registry. Adv Interv Cardiol 2016; 12: 128-34.

13. Smolka G, Pysz P, Ochała A, et al. Transcatheter paravalvular leak closure and hemolysis - a prospective registry. Arch Med Sci 2017; 13: 575-84.

14. Ozkan M, Astarcıoğlu MA, Gürsoy MO. Evaluation of endothelialization after percutaneous closure of paravalvular leaks. J Invasive Cardiol 2012; 24: E72-4. 